Research Article

Mehran Karimi Zeverdejani and Yaghoub Tadi Beni*

\title{
Size-dependent vibration analysis of graphene-PMMA lamina based on non-classical continuum theory
}

https://doi.org/10.1515/secm-2019-0033

Received Jan 28, 2019; accepted Mar 31, 2019

\begin{abstract}
This paper studies the free vibration of polymer nanocomposite reinforced by graphene sheet. In this work, the new size dependent formulation is presented for nanocomposites based on couple stress theory. For this purpose, the first shear deformation theory is applied. The effect of scale parameter is investigated based on anisotropic couple stress theory. Vibration equations of the composite lamina are extracted using Hamilton's principle. Numerical results are provided for Poly methyl methacrylate/graphene composite. Mechanical properties of the composite are obtained from molecular dynamics simulation. Based on eigenvalue procedure, an analytical solution is obtained for the natural frequency of composite lamina. In the results section, the effect of dimensional and physical parameters are investigated on lamina natural frequency. It is observed that graphene defects caused to diminish the lamina frequency. Furthermore, it is revealed that the increase in graphene volume fraction leads to natural frequency be greater.
\end{abstract}

Keywords: composite lamina, anisotropic, size effect, couple stress theory, graphene defects

\section{Introduction}

Today, composite materials have improved the performance in various mechanisms. Additionally, by exploiting the low volume fraction of the reinforcement, the mechanical properties of structures have been greatly improved and lead to the ideal efficiency of consumables [1-4]. Basically, designers are eager to build the structures with high

\footnotetext{
${ }^{\star}$ Corresponding Author: Yaghoub Tadi Beni: Faculty of Engineering, Shahrekord University, Shahrekord, Iran; Email: tadi@eng.sku.ac.ir

Mehran Karimi Zeverdejani: Mechanical engineering department, Shahrekord University, Shahrekord, Iran
}

strength to weight ratio. Depend on the applications, there are various applicable types of reinforcements and matrixes. Among the most widely used composites, one can be mentioned to metal-metal, metal-ceramics and polymer composites $[5,6]$. Including the nanoscale reinforcements, it can be noted to the graphene sheet, carbon nanotube, and fullerene which the second and third elements made from twisting and rounding the graphene sheets, respectively [7]. Graphene is one of the thinner known materials in the nature that is about 0.34 nanometers thick. Graphene has special properties compared to other nanoreinforcements. Because the top and bottom surfaces of the graphene sheet surrounded in the matrix, it has an excellent surface contacts. Moreover, the price of graphene is reasonable $[8,9]$.

Thus far, several studies have been conducted on polymer composite reinforced by nanoparticles [10-13]. Shen et al. $[14,15]$ investigated vibration and buckling of functionally graded composite reinforced by graphene and carbon nanotube (CNT). Mirzaei and Kiani [16] studied vibration of functionally graded CNT reinforced composite cylindrical panels. Zhang et al. [17] investigated the vibration of triangle composite plate reinforced by CNT.

In addition to macro, Composites are also used in micro and nano scales. For instance, nano-scale composites utilize in the production of nanoelectromechanical systems (NEMS) and microelectromechanical systems (MEMS) [18]. In the nanoscale, due to certain physical conditions and the low ratio of molecular distance to the structural dimensions, the material scale parameter affects the mechanical behavior of the structure [19]. As a result, the size effect must be applied in constitutive equations. There are several size dependent continuum theories. In some of these theories, the scale parameter has a softening effect on the results, such as nonlocal Eringen's theories [20-22]. Barretta et al. [23] presented nonlocal integral model for Timoshenko beam model. Romano et al. [24] studied constitutive boundary condition of nano-beam based on nonlocal theory. Higher order nonlocal Euler Bernoulli beam model presented by Barretta et al. [25]. Acierno et al. [26]

ð Open Access. ( 2019 M. K. Zeverdejani and Y. T. Beni, published by De Gruyter. 
measured mechanical properties of polymer nano composite based on both nonlocal theory and experimental approach. Also, the nonlocal elastoplastic model presented by De sciara [27]. In some other theories, the scale parameter has a hardening effect on the results, such as the strain gradient and couple stress theories [28-30]. Aifanties [31] presented update of scale dependent gradient theories. Reviewed study was conducted on the stress gradient theory for size-dependent response of structures by Askes and Gitman [32]. Furthermore, some studies conducted based on nonlocal strain gradient theory $[33,35]$. Barretta and Sciarra [36] investigated constitutive boundary conditions of nano- beams according to nonlocal strain gradient theory. Vibration analysis of elastic beam was studied by Apuzzu et al. [37] based on nonlocal gradient theory. Canadija et al. [38] extended nonlocal gradient formulation for beam model.

For the first time, couple stress theory was presented by Mindlin in 1962 [39]. So far, many scientists have studied the behavior of nanoscale elements using this theory [40-44]. Chen and Yang [45] developed the couple stress theory for anisotropic behavior. Some scholars using the anisotropic couple stress theory have studied the mechanical behavior of anisotropic structures. Tadi et al. [46, 47] studied free vibration and buckling of CNT based on anisotropic size dependent shell model. Gao et al. [48] benefitting anisotropic couple stress model studied the static deformation of layered magneto electroelastic plates under surface loading. Buckling analysis of orthotropic protein microtubules investigated by Tadi et al. [49]. Yang and He [50] investigated static analysis of orthotropic microplate based on modified couple stress theory. Tsiatas and Yiotis [51] studied the static, dynamic and buckling of the anisotropic skew plate. It should be noted that conventional size dependent models have been challenged in some new studies [52-54]. The kinematics of generalized micro-morphic continua is presented by Romano et al. [52]. They proposed the simplest non-redundant model by dropping the micro-curvature term from the Mindlin theory. Furthermore, Barbagallo et al. [53] showed that a general formula is not applicable for the standard Mindlin-Eringen-format of the anisotropic micro-morphic model. Furthermore, Neff et al. [54] presented the relaxed micro-morphic model and derive the set of appropriate conditions that have to be imposed on the constitutive parameters. While, some researchers used nonlocal and gradient based size dependent theories [55].

Given that mechanical testing and control of its process are very costly and complex, molecular simulation is a suitable method for determining the materials properties. Some researchers calculated mechanical properties of nanocomposites by molecular dynamics simulations (MD). Han an Eliot [56] calculated mechanical properties of polymer composite reinforced by CNT using MD simulation. Lin et al. [57] using MD obtained mechanical properties of graphene-reinforced polymer composites. Moreover, Yunlong et al. [58] studied the mechanical properties of polymer composite reinforced by CNT and graphene sheet.

In this paper, new size dependent formulation is presented for the vibrational behavior of nano-composite. For sake of this aim, the anisotropic motion equations are extracted using Hamilton's principle. These equations are developed for the first shear deformation plate model (FSDT). Furthermore, the size effect is involved according to the couple stress theory. It is noted the present vibrational equations are reduced to different states in certain conditions:

- If the mechanical properties of the plate are identical in various directions, vibration equations of the size-dependent isotropic plate are obtained.

- If the scale parameter is ignored, vibration equations of the orthotropic plate are extracted for classical continuum mechanic.

- By combining the two above assumptions, vibration equations are derived for an isotropic macro plate.

Indeed, current work presents the new formulation for orthotropic composite plates which considers orthotropic and size sensitivity behavior of composite plates, simultaneously. Mechanical properties are also evaluated based on the molecular simulation. For the case study, the vibration of all edges simply supported (SSSS) Poly methyl methacrylate (PMMA) lamina reinforced by graphene sheet are investigated. Elastic properties of lamina reinforced by defected graphene are gained by molecular dynamics simulation. In the results section, the effect of physical and geometric parameters on the natural frequency of lamina has been investigated. Also, different vibrational mode shapes of lamina have been shown.

\section{Size-dependent formulation for composite plate}

In this work, the lamina is modeled as FSDT plate. The displacement components of this model are defined as follows:

$$
\begin{aligned}
u & =u_{0}(x, y, t)+z \phi_{x}(x, y, t) \\
v & =v_{0}(x, y, t)+z \phi_{y}(x, y, t) \\
w & =w_{0}(x, y, t)
\end{aligned}
$$



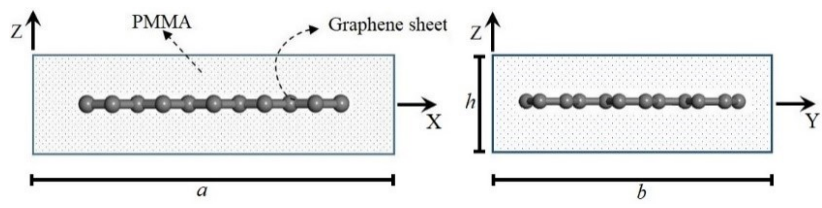

Figure 1: Schematic of the X-Z and Y-Z views of Graphene reinforced lamina.

In the above equation $u, v$ and $w$ are total displacements related to $x, y$ and $z$ directions, respectively. $u_{0}, v_{0}$, and $w_{0}$ indicate the in-plane displacements (at the $\mathrm{z}=0$ ). Also, $\phi_{x}$ and $\phi_{y}$ represent a rotation around y and x-axes. Schematic of the lamina is illustrated in Figure 1. Based on energy methods, Hamilton's principle is used to develop the motion equations. According to this theory, the variation of the system energy in a period of time is equal to zero as follows:

$$
\delta \int_{0}^{t}[T-U] d t=0
$$

In the above equation, $U$ expresses lamina strain energy, and $T$ indicates the lamina kinetic energy. Based on the couple stress theory, the strain energy of a body is obtained through the following integration:

$$
U=\frac{1}{2} \int_{\Omega}[\sigma: \varepsilon+m: \chi] d V
$$

Where $\boldsymbol{\epsilon}, \boldsymbol{\sigma}, \boldsymbol{\chi}$ and $\boldsymbol{m}$ represent strain tensor, Cauchy's stress tensor, rotation gradient tensor, and higher order stress tensor, respectively. Based on the couple stress formulation, the strain and rotation gradient tensors are defined as follows:

$$
\begin{aligned}
\boldsymbol{\varepsilon}= & \frac{1}{2}\left[\operatorname{grad}(\vec{u})+(\operatorname{grad}(\vec{u}))^{T}\right] \\
\chi & =\frac{1}{2}\left[\operatorname{grad}\left(\frac{1}{2} \operatorname{curl}(\vec{u})\right)\right. \\
& \left.+\left(\operatorname{grad}\left(\frac{1}{2} \operatorname{curl}(\vec{u})\right)\right)^{T}\right]
\end{aligned}
$$

In the following sections, the calculation process of classic and higher-order stresses using $\boldsymbol{\epsilon}$ and $\boldsymbol{\chi}$ values is explained. For orthotropic materials, the relationship between stress and strain is expressed as follows:

$$
\left\{\begin{array}{l}
\sigma_{x x} \\
\sigma_{y y} \\
\sigma_{x z} \\
\sigma_{y z} \\
\sigma_{x y}
\end{array}\right\}=\left[\begin{array}{ccccc}
Q_{11} & Q_{12} & 0 & 0 & 0 \\
Q_{21} & Q_{22} & 0 & 0 & 0 \\
0 & 0 & Q_{55} & 0 & 0 \\
0 & 0 & 0 & Q_{66} & 0 \\
0 & 0 & 0 & 0 & Q_{44}
\end{array}\right]\left\{\begin{array}{c}
\varepsilon_{x x} \\
\varepsilon_{y y} \\
\varepsilon_{x z} \\
\varepsilon_{y z} \\
\varepsilon_{x y}
\end{array}\right\}
$$

$$
=\left[\begin{array}{ccccc}
\frac{E_{x x}}{1-v_{x y} v_{y x}} & \frac{v_{x y} E_{y y}}{1-v_{x y}} & 0 & 0 & 0 \\
\frac{v_{y x} E_{x x}}{1--_{x y} v_{y x}} & \frac{E_{y y}}{1-v_{x y} v_{y x}} & 0 & 0 & 0 \\
0 & 0 & 2 G_{y z} & 0 & 0 \\
0 & 0 & 0 & 2 G_{x z} & 0 \\
0 & 0 & 0 & 0 & 2 G_{x y}
\end{array}\right]\left\{\begin{array}{c}
\varepsilon_{x x} \\
\varepsilon_{y y} \\
\varepsilon_{x z} \\
\varepsilon_{y z} \\
\varepsilon_{x y}
\end{array}\right\}
$$

Besides, the relationship between higher-order stress and rotation gradient is defined as follows [45-47]:

$$
m_{i j}=l_{i}^{2} G_{i j} \chi_{i j}+l_{j}^{2} G_{j i} \chi_{j i}
$$

In the above equation, $l_{i}$ represents the material length scale parameter. By substituted Eq. (1) into Eq. (4), components of lamina strain are derived as follows:

$$
\begin{gathered}
\varepsilon_{x x}=u_{0, x}+z \phi_{x, x} \\
\varepsilon_{y y}=v_{0, y}+z \phi_{y, y} \\
\varepsilon_{x z}=\frac{1}{2}\left(\phi_{x}+w_{0, x}\right) \\
\varepsilon_{x y}=\frac{1}{2}\left(v_{0, x}+u_{0, y}+z\left(\phi_{y, x}+\phi_{x, y}\right)\right) \\
\varepsilon_{y z}=\frac{1}{2}\left(\phi_{y}+w_{0, y}\right)
\end{gathered}
$$

As well as, by substituting Eq. (1) into Eq. (5), components of lamina rotation gradient tensor are obtained as follows:

$$
\begin{gathered}
\chi_{x x}=\frac{1}{2}\left[w_{0, x y}-\phi_{y, x}\right] \\
\chi_{y y}=\frac{1}{2}\left[\phi_{x, y}-w_{0, x y}\right] \\
\chi_{z z}=\frac{1}{2}\left[\phi_{y, x}-\phi_{x, y}\right] \\
\chi_{x y}=\frac{1}{4}\left[w_{0, y y}-\phi_{y, y}+\phi_{x, x}-w_{0, x x}\right] \\
\chi_{x z}=\frac{1}{4}\left[v_{0, x x}-u_{0, x y}+z \phi_{y, x x}-z \phi_{x, x y}\right] \\
\chi_{z y}=\frac{1}{4}\left[v_{0, x y}-u_{0, y y}+z \phi_{y, x y}-z \phi_{x, y y}\right]
\end{gathered}
$$

According to Eqs. (3), (6) and (7), and by using components of strain and rotation gradient tensors which were obtained in the Eqs. (8) to (18), the strain energy of composite is obtained as follows:

$$
U=\iiint_{V}\left\{\frac{E_{x x}}{1-v_{x y} v_{y x}}\left(u_{0, x}+z \phi_{x, x}\right)^{2}\right.
$$


$+\frac{v_{x y} E_{y y}}{1-v_{x y} v_{y x}}\left(u_{0, x}+z \phi_{x, x}\right)\left(v_{0, y}+z \phi_{y, y}\right)$

$+\frac{E_{y y}}{1-v_{x y} v_{y x}}\left(v_{0, y}+z \phi_{y, y}\right)^{2}$

$+\frac{v_{y x} E_{x x}}{1-v_{x y} v_{y x}}\left(u_{0, x}+z \phi_{x, x}\right)\left(v_{0, y}+z \phi_{y, y}\right)$

$+G_{x z}\left(\phi_{x}+w_{0, x}\right)^{2}+G_{x y}\left(v_{0, x}+u_{0, y}+z\left(\phi_{y, x}+\phi_{x, y}\right)\right)^{2}$

$+G_{y z}\left(\phi_{y}+w_{0, y}\right)^{2}+\frac{1}{2} l_{x}^{2} G_{x x}\left(w_{0, x y}-\phi_{y, x}\right)^{2}$

$+\frac{1}{2} l_{y}^{2} G_{y y}\left(\phi_{x}, y-w_{0, x y}\right)^{2}$

$+\frac{1}{8} G_{x y}\left(w_{0, y y}-\phi_{y, y}+\phi_{x, x}-w_{0, x x}\right)^{2}\left(l_{x}^{2}+l_{y}^{2}\right)$

$+\frac{1}{2} l_{z}^{2} G_{z z}\left(\phi_{y, x}-\phi_{x}, y\right)^{2}$

$+\frac{1}{8} G_{x z}\left(v_{0, x x}-u_{0, x y}+z \phi_{y, x x}-z \phi_{x, x y}\right)^{2}\left(l_{x}^{2}+l_{z}^{2}\right)$

$+\frac{1}{8} G_{y z}\left(v_{0, x y}-u_{0, y y}+z \phi_{y, x y}\right.$

$\left.\left.-z \phi_{x, y y}\right)^{2}\left(l_{z}^{2}+l_{y}^{2}\right)\right\} d V$

After determining the lamina strain energy, the kinetic energy of composite is calculated. Using the time derivative on the FSDT displacement vector, the plate kinetic energy is computed as follows:

$$
\begin{aligned}
T & =\frac{\rho}{2} \iiint_{V}\left\{u_{0, t}^{2}+z^{2} \phi_{x, t}^{2}+2 z \phi_{x, t} u_{0, t}+v_{0, t}{ }^{2}\right. \\
& \left.+z^{2} \phi_{y, t}^{2}+2 z \phi_{y, t} v_{0, t}+w_{0, t}^{2}\right\} d V
\end{aligned}
$$

By substituting Eqs. (19) and (20) into Eq. (2), and using calculus of variation approach, the size-dependent vibration equations of the orthotropic lamina are developed as follows:

$$
\begin{aligned}
& C_{1} u_{0, x x}+C_{2} v_{0, x y}+C_{3} u_{0, y y}+C_{4} v_{0, x x x y}+C_{5} u_{0, x x y y} \\
& +C_{6} v_{0, x y y y}+C_{7} u_{0, y y y y}+C_{8} u_{0, t t}=0 \\
& C_{9} v_{0, y y}+C_{10} u_{0, x y}+C_{11} v_{0, x x}+C_{12} v_{0, x x x x} \\
& +C_{13} u_{0, x x x y}+C_{14} v_{0, x x y y}+C_{15} u_{0, x y y y}+C_{16} v_{0, t t}=0 \\
& C_{17} \phi_{x, x}+C_{18} w_{0, x x}+C_{19} \phi_{y, y}+C_{20} w_{0, y y} \\
& +C_{21} w_{0, x x y y}+C_{22} \phi_{y, x x y}+C_{23} \phi_{x, x y y}+C_{24} \phi_{y, y y y} \\
& +C_{25} w_{0, y y y y}+C_{26} \phi_{x, x x x}+C_{27} w_{0, x x x x}+C_{28} w_{0, t t}=0 \\
& C_{29} \phi_{x, x x}+C_{30} \phi_{y, x y}+C_{31} \phi_{x, y y}+C_{32} \phi_{x}+C_{33} w_{0, x} \\
& +C_{34} w_{0, x y y}+C_{35} w_{0, x x x}+C_{36} \phi_{y, x x x y}+C_{37} \phi_{x, x x y y} \\
& +C_{38} \phi_{y, x y y y}+C_{39} \phi_{x, y y y y}+C_{40} \phi_{x, t t}=0 \\
& C_{41} \phi_{y, y y}+C_{42} \phi_{x, x y}+C_{43} \phi_{y, x x}+C_{44} \phi_{y}+C_{45} w_{0, y}
\end{aligned}
$$

$$
\begin{aligned}
& +C_{46} w_{0, x x y}+C_{47} w_{0, y y y}+C_{48} \phi_{y, x x x x}+C_{49} \phi_{x, x x x y} \\
& +C_{50} \phi_{y, x x y y}+C_{51} \phi_{x, x y y y}+C_{52} \phi_{y, t t}=0
\end{aligned}
$$

In the above equations, $C_{i j}$ 's are reliant on the mechanical properties of the lamina which provided in appendix A.

\section{Molecular dynamics simulation}

This section may be divided by subheadings. It should provide a concise and precise description of the experimental results, their interpretation as well as the experimental conclusions that can be drawn. As mentioned in the introduction section, use of the experimental procedure for extract the mechanical properties of nanoscale composites are very complicated and difficult, and one of the most suitable methods is use of molecular dynamics. The molecular dynamics method is one of the powerful methods of molecular mechanics which is designed based on numerical integration of Newton's equations on the molecular domain of a body. In order to perform molecular dynamics simulation, first, the initial conditions of the system including the speed and the primary location of the particles are determined. Then, by selecting a suitable time interval, the new atomic positions will be predicted according to the previous position and initial velocity. Intermolecular interactions using a function the potential is expressed. In fact, the potential function is the main input in the molecular dynamics simulation. The potential function of $\mathrm{N}$-particle systems is given by the following equation:

$$
\begin{aligned}
\phi(r) & =\sum_{i} \phi_{1}\left(r_{i}\right)+\sum_{i} \sum_{j\rangle i} \phi_{2}\left(r_{i}, r_{j}\right) \\
& +\sum_{i} \sum_{j>i} \sum_{k>j} \phi_{3}\left(r_{i}, r_{j}, r_{k}\right)+\ldots
\end{aligned}
$$

Where $\phi_{i}$ is represented the various kind of interactions for individual particles. The force applied to each particle is calculated from the following equation:

$$
F_{i}=\frac{\partial \phi}{\partial r_{i}}
$$

Also, according to Newton's second law, the following basic relation is extracted for each particle

$$
F_{i}=\frac{\partial \phi}{\partial r_{i}}=m_{i} \ddot{r}_{i}
$$

Using the above equation, we can calculate the acceleration of each particle and then using numerical integration, in a very small time interval, the new position and speed of the particles have been achieved. In the present 


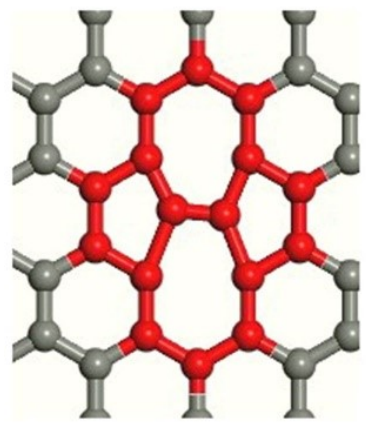

(a)

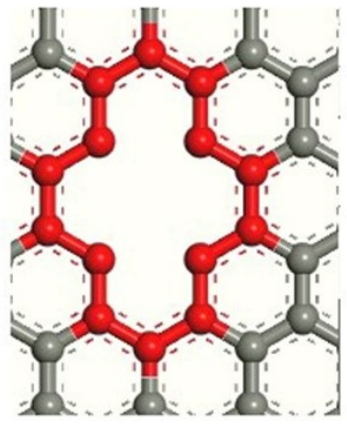

(b)

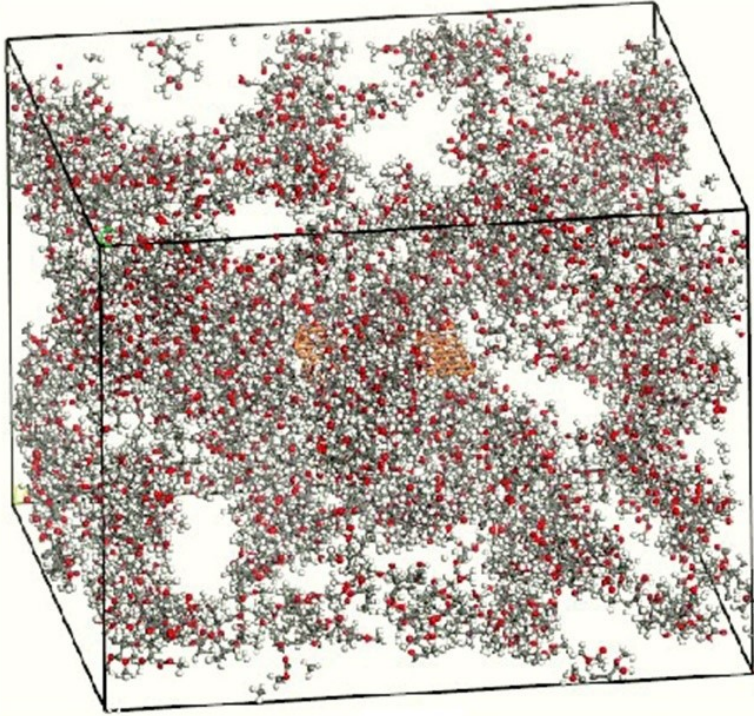

(c)

Figure 2: (a) Stone Walls defect (SW), (b) Vacancy Defect (VD), (c) molecular model of the lamina.

Table 1: Mechanical properties of the composite with 0.19 volume fraction for various graphene defects.

\begin{tabular}{cccc}
\hline Type of graphene-reinforced in PMMA & $\mathbf{E}_{\mathbf{1 1}}(\mathbf{G P a})$ & $\mathbf{E}_{\mathbf{2 2}}(\mathbf{G P a})$ & $\mathbf{G}_{\mathbf{1 2}}(\mathbf{G P a})$ \\
\hline Perfect graphene & 104.1 & 107.7 & 22.7 \\
Graphene with 1.6\% vacancy & 98.1 & 100.5 & 20.8 \\
Graphene with 5\% vacancy & 93.2 & 92.6 & 18.8 \\
Graphene with 1.6\% stone wales & 103.5 & 97 & 21.4 \\
Graphene with 5\% stone wales & 91.9 & 86.9 & 19.7 \\
\hline
\end{tabular}

work, the mechanical properties of the composite are calculated using Material Studio software and effects of vacancy (VD) and stone wales (SW) defects of graphene are explored on the stiffness of lamina. The stone wales and vacancy defects along the molecular model of the lamina are shown in Figure 2. Composite is modeled based on COMPASS forcefield. In the beginning, the molecular model of the lamina is subjected to NVT ensemble in $450^{\circ} \mathrm{K}$. Then, it is exposed to NPT ensemble by $0.1 \mathrm{GPa}$ pressure. In the end, by applying finite strain, mechanical properties of the lamina are determined according to Table 1.

\section{Solution method}

Based on Navier's solution method, for simply supported lamina, the displacement components are considered as follows:

$$
u_{0}=A_{1} \cos \left(\frac{m \pi x}{L}\right) \cos \left(\frac{m \pi y}{L}\right) e^{i \omega t}
$$

$$
\begin{aligned}
v_{0} & =A_{2} \sin \left(\frac{m \pi x}{L}\right) \sin \left(\frac{m \pi y}{L}\right) e^{i \omega t} \\
w_{0} & =A_{3} \sin \left(\frac{m \pi x}{L}\right) \cos \left(\frac{m \pi y}{L}\right) e^{i \omega t} \\
\phi_{x} & =A_{4} \cos \left(\frac{m \pi x}{L}\right) \cos \left(\frac{m \pi y}{L}\right) e^{i \omega t} \\
\phi_{y} & =A_{5} \sin \left(\frac{m \pi x}{L}\right) \sin \left(\frac{m \pi y}{L}\right) e^{i \omega t}
\end{aligned}
$$

In the above equation, $\omega$ is the lamina natural frequency. Also, $A_{1}, A_{2}, A_{3}, A_{4}$, and $A_{5}$ represent the vibration amplitude for each of the variables. Moreover, $m$ and $\mathrm{n}$ represent the longitudinal mode number and circumferential mode number, respectively. By substituting Eq. (29) into the motion equations, the resulted equations are converted to following eigenvalue problem:

$$
\left([K]-\left[M \omega^{2}\right]\right)\left\{A_{i}\right\}=0
$$

In the above equation, $\mathrm{K}$ and $\mathrm{M}$ are stiffness and mass matrixes. After solving Eq. (30), the lamina natural frequency is obtained, which will be discussed in detail in the results section. 

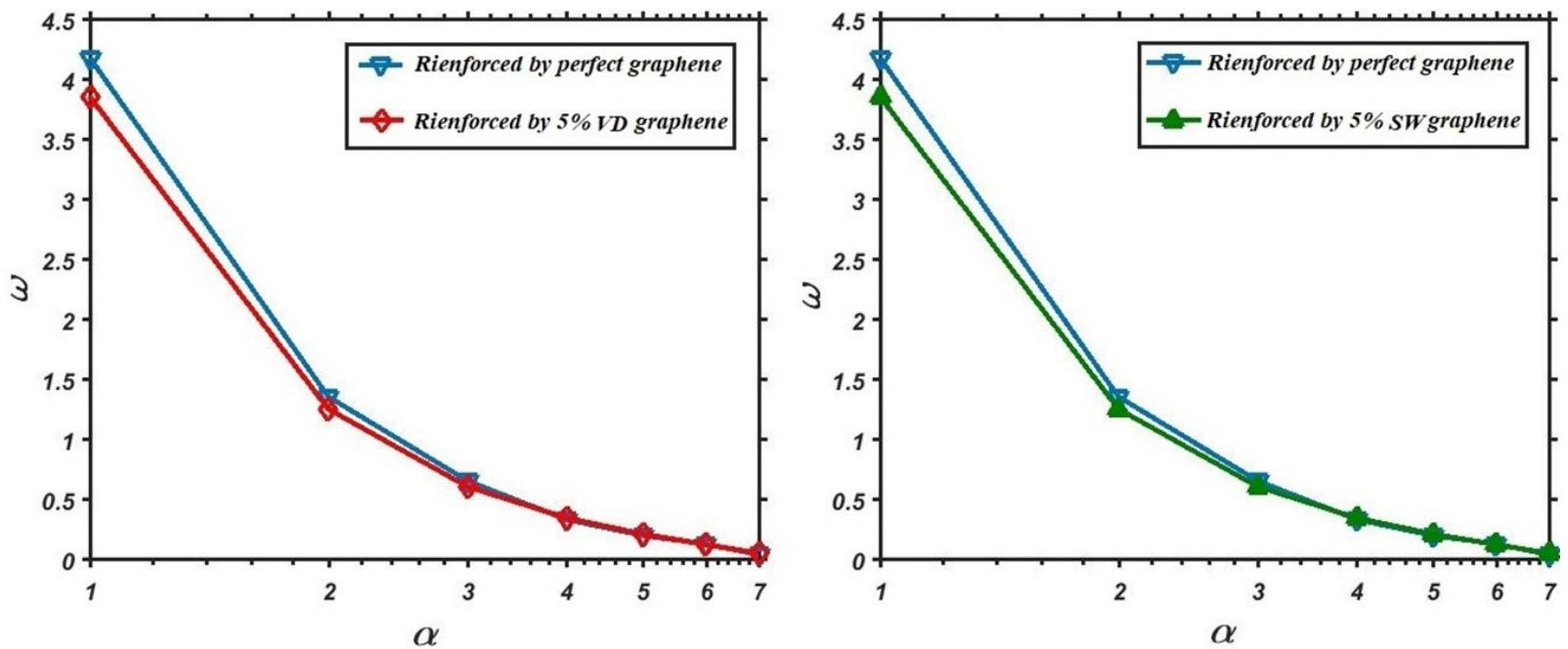

Figure 3: Variation of lamina frequency with $\alpha$ parameter: a) vacancy defect b) stone wales defect.

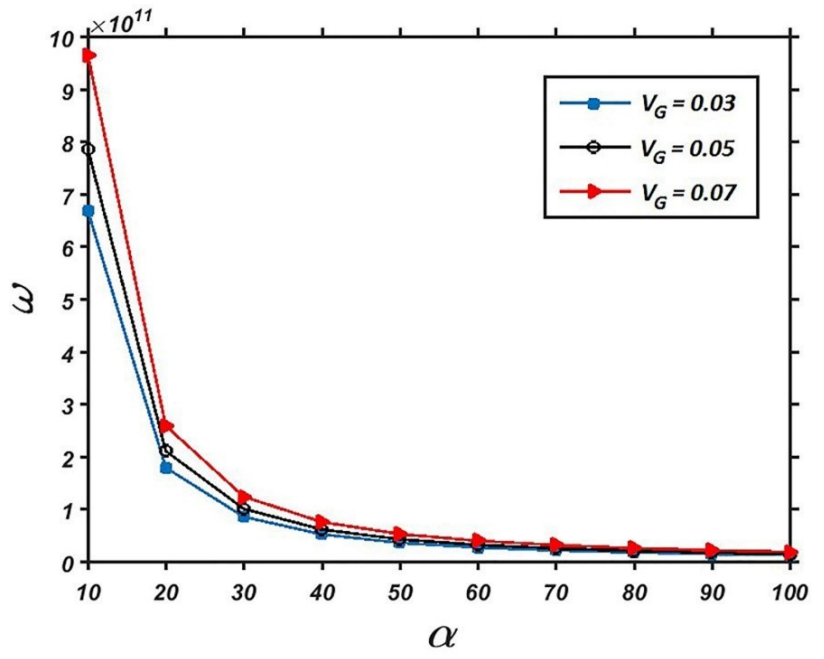

Figure 4: Variation of lamina natural frequency with $\alpha$ parameter for different graphene volume fraction.

\section{Results and discussions}

The aim beyond of the present work is to study the free vibration of Graphene-reinforced lamina. Predict the oscillation behavior of nano-structures can be useful for the control of their performance. Polymeric composites reinforced by graphene sheets have a good physical properties and can be used in nanoelectromechanical systems. In this section, according to the procedure that presented before, the vibration of simply supported lamina composite is investigated using the orthotropic FSDT model. Various numerical examples are proposed to investigate the influence of geometrical and material properties on the vibration behavior of lamina. In order to study the vibrational behavior of current composite model, natural frequencies are presented for the various geometrical parameters, $\alpha$ parameter represents ratio of length to lamina thickness $(a / h) . \beta$ parameter also signifies width to lamina thickness $(b / h)$. Furthermore, $\lambda$ parameter is defined as a non-dimensional length scale parameter $(l / h)$. Besides, the material length scale parameter is assumed as $l_{1}=l_{2}=l_{3}=l$. By literature review, the value of length scale $l$ is calibrated for graphene sheet by $2.46 \mathrm{~nm}$ length and width. Arash and Wang [59] using MD simulation demonstrated that the natural frequency of the aforementioned graphene is equal to $77.6 \mathrm{GHz}$. By comparison this frequency with the solution of equation (30), length scale parameter is calibrated in 0.118 nm.

In the Figure 3, influences of graphene defects on lamina natural frequency are studied. The mechanical properties of the composites reinforced by defected graphene are given in Table 1. According to the following graphs, it is clear that vacancy (VD) and stone wales (SW) defects lead to decrease lamina frequency. Furthermore, it is obvious that the impact of graphene defects is more severe for lower $\alpha$ parameter.

In Figure 4, the variation of the lamina frequency related to $\alpha$ parameter is shown. As seen in this figure, by increasing of $\alpha$ parameter lamina natural frequency is reduced. It is also clear that by increasing the volume fraction of graphene, the natural frequency of lamina has grown that for $\alpha$ smaller than 60 this increase is more significant. It should be noted mechanical properties for the various volume fraction of reinforcement have been used from Shen study [40].

In Figure 5 the simultaneous effects of $\alpha$ and $\beta$ dimensionless parameters are illustrated. According to this fig- 


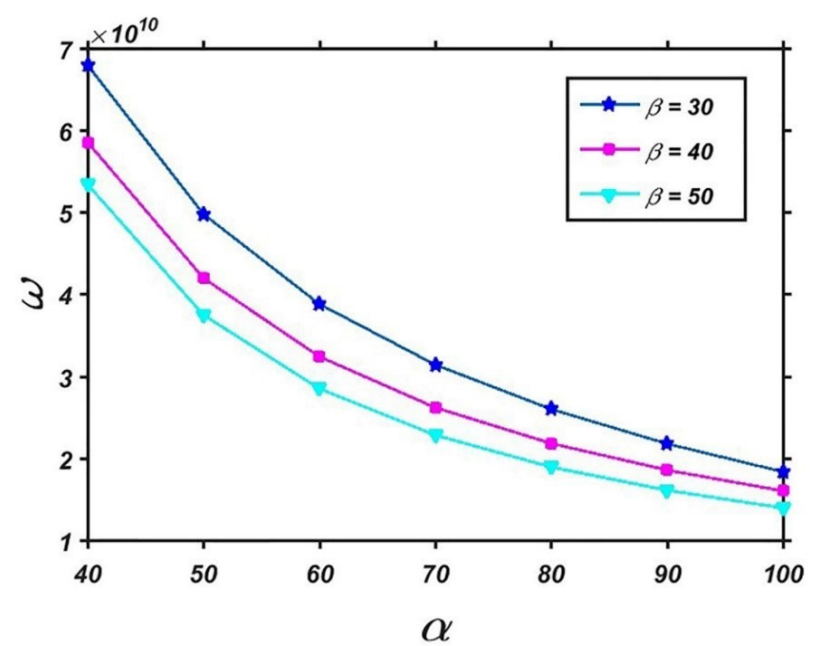

Figure 5: Variation of the natural frequency with $\alpha$ for different $\beta$ parameters.

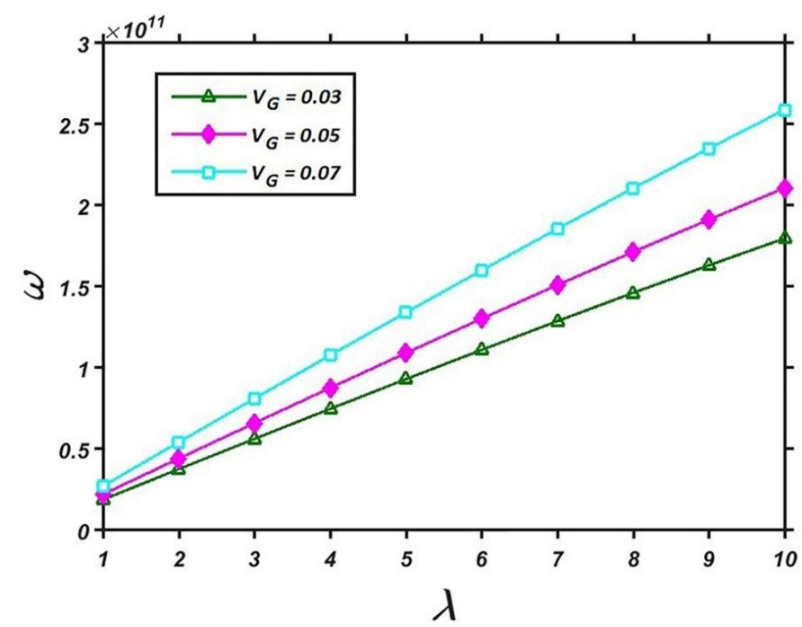

Figure 6: Variation of the natural frequency with $\lambda$ for different graphene volume fraction.

ure, the highest lamina natural frequency has been obtained for smaller $\alpha$ and $\beta$ parameters.

In Figure 6, the effect of the material length scale parameter is investigated on the results. It is observed that by increasing in $\lambda$ parameter vibrational frequency of lamina increases which this result confirms the hardening effect of couple stress scale parameter. In addition, it is observed that the rate of frequency increase is more severe for composites with higher volume fractions of the graphene. Figure 7 , provides the various vibration mode shapes of the lamina. In this figure, different mode shapes along with their corresponding frequencies are observed which $\alpha=$ $50, \beta=50, \lambda=1$ and $V_{G}=0.03$ are considered.

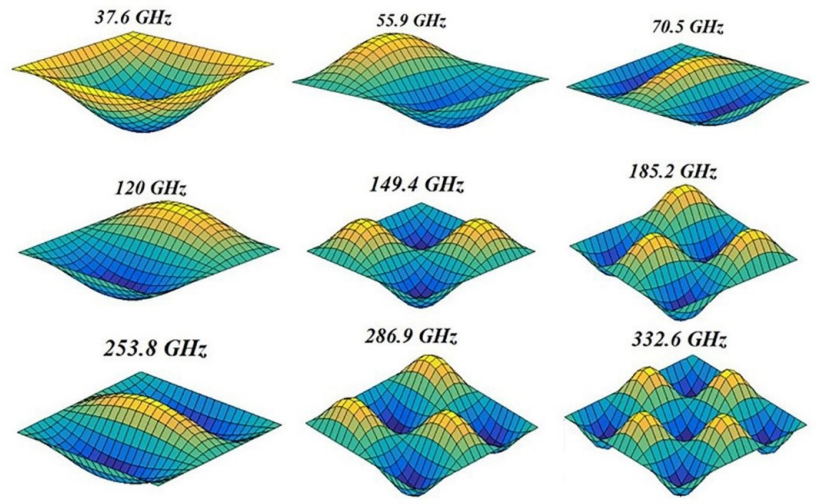

Figure 7: Various vibrational mode shapes of the lamina.

\section{Conclusion}

In this paper, a new model was presented for nanoscale composites. For this purpose, the composite was modeled as FSDT plate model. Scale sensitivity was considered according to couple stress theory. Using the variations principles governing equations of anisotropic plate were derived. In order to a case study, free vibration of graphenereinforced polymer lamina was examined. Mechanical properties of lamina reinforced by defected graphene were obtained by molecular dynamic simulation.

After solving the lamina free vibration equations, the results demonstrated that the increase in graphene volume fraction leads to increase in lamina frequency. Furthermore, it was determined that although the graphene volume fraction increases composite frequency, the intensity of the effect of this parameter for lamina with $\alpha>0.01$ condition is stronger. In addition, it was founded that the impact of raising the scale parameter on the lamina frequency is stronger for higher graphene volume fraction.

\section{References}

[1] Kaw, Autar K. mechanic of composite materials. s.l. : CRC Press, 2006.

[2] Globus, A., Bailey, D., Han, J., Jaffe, R., Levit, C., Merkle, R.Srivastava, D. Nasa applications of molecular nanotechnology, 1998.

[3] Silvia G. Prolongo, Adam D. Printz, Nicholas Rolston, Brian L. Watson, Reinhold H. Dauskardt, Poly(triarylamine) composites with carbon nanomaterials for highly transparent and conductive coatings, Thin Solid Films, 646, Pages 61-66, 2018.

[4] Paula, D.R, Robeson, L.M.Polymer nanotechnology: Nanocomposites, Polymer, Vol. 49, pp. 3187-3204, 2008.

[5] Roghayeh Ghasempour, Hamid Narei, CNT Basics and Characteristics, Carbon Nanotube-Reinforced Polymers, 1-24, 2018. 
[6] Zeynel Ozturk, Cengiz Baykasoglu, Mesut Kirca, Sandwiched graphene-fullerene composite: A novel 3-D nanostructured material for hydrogen storage, International Journal of Hydrogen Energy, Volume 41, 6403-6411, 2016.

[7] Kuilla, T, Bhadra, S. Recent advances in graphene based polymer composites, Progress in Polymer Science, Vol. 35, pp. 1350-1375, 2010.

[8] Vijay Ponraj, N, Azhagurajan, A. Graphene nanosheet as reinforcement agent in copper matrix composite by using powder metallurgy method, Surfaces, and Interfaces, Vol. 6, pp. 190196, 2017.

[9] Lin, F, Xiang, Y, Shen, H.S. Temperature dependent mechanical properties of graphene-reinforced polymer nanocomposites - a molecular dynamics simulation. 2017, Compos. Part B, Vol. 111, pp. 261-269.

[10] Anisha Christy, Rajesh Purohit, R.S. Rana, Swadesh Kumar Singh, Saraswati Rana, Development and Analysis of Epoxy/nano SiO2 Polymer Matrix Composite fabricated by Ultrasonic Vibration assisted Processing, Materials Today: Proceedings, Volume 4, Part A, 2748-2754, 2017.

[11] Yuanliang Zhao, Xiaowen Qi, Yu Dong, Jian Ma, Qingxiang Yang, Mechanical, thermal and tribological properties of polyimide/nano-SiO2 composites synthesized using an in-situ polymerization, Tribology International, Volume 103, 599-608, 2016.

[12] Mitao Song, Jie Yang, Sritawat Kitipornchai, Bending and buckling analyses of functionally graded polymer composite plates reinforced with graphene nanoplatelets, Composites Part B: Engineering, Volume 134, 106-113, 2018.

[13] Alireza Gharib, Mohammad Saeed Karimi, Ali Ghorbanpour Arani, Vibration analysis of the embedded piezoelectric polymeric nanocomposite panels in the elastic substrate, Composites Part B: Engineering, Volume 101, 64-76, 2016.

[14] Hui-Shen Shen, Y. Xiang, Feng Lin, D. Hui, Buckling and postbuckling of functionally graded graphene-reinforced composite laminated plates in thermal environments, Composites Part B: Engineering, Volume 119, 67-78, 2017.

[15] Hui-Shen Shen, Y. Xiang, Feng Lin, Nonlinear vibration of functionally graded graphene-reinforced composite laminated plates in thermal environments, Computer Methods in Applied Mechanics and Engineering, Volume 319,175-193, 2017.

[16] M. Mirzaei, Y. Kiani, Free vibration of functionally graded carbon nanotube reinforced composite cylindrical panels, Composite Structures, Volume 142, 45-56, 2016.

[17] L.W. Zhang, Z.X. Lei, K.M. Liew, Free vibration analysis of functionally graded carbon nanotube reinforced composite triangular plates using the FSDT and element-free IMLS Ritz method, Composite Structures, Volume 120, 189-199, 2015.

[18] Dongzhi Zhang, Tianhong Cui, Tunable mechanical properties of layer-by-layer self-assembled carbon nanotube/polymer nanocomposite membranes for M/NEMS, Sensors and Actuators A: Physical, Volume 185, 101-108, 2012.

[19] Tadi Beni, Y., Karimi Zeverdejani, M. Free vibration of microtubules as elastic shell model based on modified couple stress theory. Journal of Mechanics in Medicine and Biology, 15, 1550037, 2015.

[20] Y. Gao, L. An, A nonlocal elastic anisotropic shell model for microtubule buckling behaviors in cytoplasm, Physica E, 42, 24062415, 2010.
[21] Shen HS, Nonlocal shear deformable shell model for bending buckling of microtubules embedded in an elastic medium, Phys Lett A. 374, 4030-4039, 2010.

[22] Zeighampour, Hamid, Yaghoub Tadi Beni, and Iman Karimipour. "Material Length Scale and Nonlocal Effects on the Wave Propagation of Composite Laminated Cylindrical Micro/Nanoshells." The European Physical Journal Plus 132, no. 12, 503, 2017.

[23] Barretta, R., Luciano, R., Marotti de Sciarra, F., \& Ruta, G. Stressdriven nonlocal integral model for Timoshenko elastic nanobeams. European Journal of Mechanics - A/Solids, 72, 275-286, 2018.

[24] Romano, G., Barretta, R., Diaco, M., \& Marotti de Sciarra, F. Constitutive boundary conditions and paradoxes in nonlocal elastic nanobeams. International Journal of Mechanical Sciences, 121, 2017.

[25] Barretta, Raffaele, Marko Čanadija, and Francesco Marotti de Sciarra. "A Higher-Order Eringen Model for Bernoulli-Euler Nanobeams." Archive of Applied Mechanics 86, no. 3, 483-495, 2016.

[26] Acierno, S., Barretta, R., Luciano, R., Marotti de Sciarra, F., \& Russo, P. Experimental evaluations and modeling of the tensile behavior of polypropylene/single-walled carbon nanotubes fibers. Composite Structures, 174, 12-18, 2017.

[27] Marotti de Sciarra, F. Novel variational formulations for nonlocal plasticity. International Journal of Plasticity, 25(2), 302-331, 2009.

[28] Shengqi Yang, Wanji Chen, On hypotheses of composite laminated plates based on new modified couple stress theory, Composite Structures 133, 46-53, 2015.

[29] Hamid Zeighampour, Yaghoub Tadi Beni, A shear deformable cylindrical shell model based on couple stress theory, Arch Appl Mech. 85, 539-553, 2015.

[30] Zeverdejani MK, Tadi Beni Y, The nano scale vibration of protein microtubules based on modified strain gradient theory, Current Appl Phys 13:1566-1576, 2013.

[31] Aifantis, E. Update on a Class of Gradient Theories. Vol. 35, 2003.

[32] Askes, Harm, and Inna M. Gitman. "Review and Critique of the Stress Gradient Elasticity Theories of Eringen and Aifantis." In Mechanics of Generalized Continua: One Hundred Years after the Cosserats, edited by Gérard A. Maugin and Andrei V. Metrikine, 203-10. New York, NY: Springer New York, 2010

[33] Mehralian, Fahimeh, Yaghoub Tadi Beni, and Mehran Karimi Zeverdejani. "Calibration of Nonlocal Strain Gradient Shell Model for Buckling Analysis of Nanotubes Using Molecular Dynamics Simulations." Physica B: Condensed Matter 521, 102-11, 2017.

[34] Mehralian, Fahimeh, Yaghoub Tadi Beni, and Mehran Karimi Zeverdejani. "Nonlocal Strain Gradient Theory Calibration Using Molecular Dynamics Simulation Based on Small Scale Vibration of Nanotubes." Physica B: Condensed Matter 514, 61-69, 2017.

[35] Yaghoub Tadi Beni , Fahimeh Mehralian, A Nonlocal Strain Gradient Shell Model for Free Vibration Analysis of Functionally Graded Shear Deformable Nanotubes, International Journal Of Engineering \& Applied Sciences, 9, 88-102, 2017

[36] Barretta, Raffaele, and Francesco Marotti de Sciarra. "Constitutive Boundary Conditions for Nonlocal Strain Gradient Elastic Nano-Beams." International Journal of Engineering Science 130, 187-198, 2018.

[37] Apuzzo, A., R. Barretta, S. A. Faghidian, R. Luciano, and F. Marotti de Sciarra. "Free Vibrations of Elastic Beams by Modified Nonlocal Strain Gradient Theory." International Journal of Engineering 
Science 133, 99-108, 2018.

[38] Čanađija, Marko, Raffaele Barretta, and Francesco Marotti de Sciarra. "A Gradient Elasticity Model of Bernoulli-Euler Nanobeams in Non-Isothermal Environments." European Journal of Mechanics - A/Solids 55, 243-255, 2016.

[39] Mindlin RD and Tiersten HF. Effects of couple-stresses in linear elasticity. Arch Ration Mech Anal; 11: 415-448, 1962.

[40] Hamed Razavi, Asghar Faramarzi Babadi, Yaghoub Tadi Beni, Free vibration analysis of functionally graded piezoelectric cylindrical nanoshell based on consistent couple stress theory, Composite Structures, Volume 160, 1299-1309, 2017.

[41] Zeighampour $\mathrm{H}$ and Tadi Beni Y. A shear deformable conical shell formulation in the framework of couple stress theory, Acta Mech. 226: 2607-2629, 2015.

[42] Tadi Beni Y, Koochi A, and Abadyan M. Using modified couple stress theory for modeling the size dependent pull-in instability of torsional nano-mirror under Casimir force. Int J Optomechatronics 2014; 8: 47-71

[43] Al-Basyouni KS, Tounsi A and Mahmoud SR. Size dependent bending and vibration analysis of functionally graded micro beams based on modified couple stress theory and neutral surface position. Compos Struct 2015; 125: 621-630.

[44] Jianshi Fang, Jianping Gu, Hongwei Wang Size-dependent threedimensional free vibration of rotating functionally graded $\mathrm{mi}$ crobeams based on a modified couple stress theory, International Journal of Mechanical Sciences, Volume 136, Pages 188199, 2018.

[45] Shengqi Yang, Wanji Chen, On hypotheses of composite laminated plates based on new modified couple stress theory, Composite Structures 133, 46-53, 2015.

[46] Y Tadi Beni, F Mehralian, M Karimi Zeverdejani, Free vibration of anisotropic single-walled carbon nanotube based on couple stress theory for different chirality, Journal of Low Frequency Noise, Vibration and Active Control 36 (3), 277-293, 2017.

[47] YT Beni, F Mehralian, MK Zeverdejani, Size-dependent buckling analysis of different chirality SWCNT under combined axial and radial loading based on orthotropic model, Materials Research Express 4 (6), 065004, 2017.

[48] Junhong Guo, Jiangyi Chen, Ernian Pan, Static deformation of anisotropic layered magnetoelectroelastic plates based on modified couple-stress theory, Composites Part B: Engineering, Volume 107, 84-96, 2016.

[49] YT Beni, MK Zeverdejani, F Mehralian, Buckling analysis of orthotropic protein microtubules under axial and radial compression based on couple stress theory, Mathematical Biosciences 292, 18-29, 2017.

[50] Zihao Yang, Dan He, Vibration and buckling of orthotropic functionally graded micro-plates on the basis of a re-modified couple stress theory, Results in Physics, Volume 7, 3778-3787, 2017.

[51] George C. Tsiatas, Aristophanes J. Yiotis, Size effect on the static, dynamic and buckling analysis of orthotropic Kirchhoff-type skew micro-plates based on a modified couple stress theory: comparison with the nonlocal elasticity theory, Acta Mechanica, 226, pp 1267-1281, 2015.

[52] Romano, Giovanni, Raffaele Barretta, and Marina Diaco. "Micromorphic Continua: Non-Redundant Formulations." Continuum Mechanics and Thermodynamics 28, no. 6, 1659-1670, 2016.

[53] Barbagallo, Gabriele, Angela Madeo, Marco Valerio d'Agostino, Rafael Abreu, Ionel-Dumitrel Ghiba, and Patrizio Neff. "Transparent Anisotropy for the Relaxed Micromorphic Model: Macro- scopic Consistency Conditions and Long Wave Length Asymptotics." International Journal of Solids and Structures 120, 7-30, 2017.

[54] Neff, Patrizio et al. "Real wave propagation in the isotropicrelaxed micromorphic model"Proceedings. Mathematical, physical, and engineering sciences, 473,2197, 20160790, 2017.

[55] Sourki, R., and S. A. Hosseini. "Coupling Effects of Nonlocal and Modified Couple Stress Theories Incorporating Surface Energy on Analytical Transverse Vibration of a Weakened Nanobeam." The European Physical Journal Plus 132, no. 4, 184, 2017.

[56] Han, Y, Elliott, J., Molecular dynamics simulations of the elastic properties of polymer/carbon nanotube composites, Computational Materials Science, Vol. 39, pp. 315-323. 2007.

[57] Lin, F, Xiang, Y, Shen, H.S. Temperature dependent mechanical properties of graphene reinforced polymer nanocomposites - a molecular dynamics simulation. 2017, Compos. Part B, Vol. 111, pp. 261-269.

[58] Yunlong Li, Shijie Wang, Quan Wang, Malcolm Xing, A comparison study on mechanical properties of polymer composites reinforced by carbon nanotubes and graphene sheet, Composites Part B: Engineering, Volume 133, Pages 35-41, 2018.

[59] Behrouz Arash, Quan Wang, Vibration of Single-and DoubleLayered Graphene Sheets, J. Nanotechnol. Eng. Med, 2(1), 011012, 2011.

\section{Appendix A}

$$
\begin{gathered}
C_{1}=-Q_{11} h \\
C_{2}=-\left(Q_{12}+Q_{66} l_{z}^{2}\right) h \\
C_{3}=-Q_{66} h \\
C_{4}=\frac{G_{x z} h\left(l_{z}^{2}+l_{x}^{2}\right)}{8} \\
C_{5}=-C_{4} \\
C_{6}=-\frac{G_{y z} h\left(l_{y}^{2}+l_{z}^{2}\right)}{8} \\
C_{7}=-C_{6} \\
C_{8}=\rho h \\
C_{9}=-Q_{22} h \\
C_{10}=C_{2}
\end{gathered}
$$




$$
\begin{aligned}
& C_{11}=C_{3} \\
& C_{30}=\frac{h}{2}\left(\frac{G_{x y}\left(l_{x}^{2}+l_{y}^{2}\right)}{4}+G_{z z} l_{z}^{2}-\frac{Q_{12} h^{2}}{6}-\frac{Q_{66} h^{2}}{6}\right) \\
& C_{12}=-C_{4} \\
& C_{13}=C_{4} \\
& C_{31}=\frac{h}{2}\left(G_{z z} l_{z}^{2}+G_{y y} l_{y}^{2}+\frac{Q_{66} h^{2}}{6}\right) \\
& C_{14}=C_{7} \\
& C_{32}=Q_{44} h \\
& C_{15}=C_{6} \\
& C_{33}=C_{32} \\
& C_{16}=C_{8} \\
& C_{34}=\frac{h}{2}\left(G_{y y} l_{y}^{2}-\frac{G_{x y}\left(l_{x}^{2}+l_{y}^{2}\right)}{4}\right) \\
& C_{17}=-Q_{44} h \\
& C_{18}=C_{17} \\
& C_{35}=\frac{G_{x y} h\left(l_{x}^{2}+l_{y}^{2}\right)}{8} \\
& C_{19}=Q_{55} h \\
& C_{36}=-\frac{G_{x z} h^{3}\left(l_{x}^{2}+l_{z}^{2}\right)}{96} \\
& C_{20}=C_{19} \\
& C_{37}=-C_{36} \\
& C_{21}=\frac{h}{2}\left(G_{x x} l_{x}^{2}+G_{y y} l_{y}^{2}-\frac{G_{x y}\left(l_{x}^{2}+l_{y}^{2}\right)}{2}\right) \\
& C_{38}=-\frac{G_{y z} h^{3}\left(l_{y}^{2}+l_{z}^{2}\right)}{96} \\
& C_{22}=\frac{h}{2}\left(\frac{G_{x y}\left(l_{x}^{2}+l_{y}^{2}\right)}{2}-G_{x x} l_{x}^{2}\right) \\
& C_{39}=-C_{38} \\
& C_{40}=\frac{\rho h^{3}}{12} \\
& C_{23}=\frac{h}{2}\left(\frac{G_{x y}\left(l_{x}^{2}+l_{y}^{2}\right)}{2}-G_{y y} l_{y}^{2}\right) \\
& C_{41}=-\frac{h}{4}\left(\frac{Q_{22} h^{2}}{3}+\frac{G_{x y}\left(l_{x}^{2}+l_{y}^{2}\right)}{2}\right) \\
& C_{24}=\frac{G_{x y} h\left(l_{x}^{2}+l_{y}^{2}\right)}{8} \\
& C_{25}=-C_{24} \\
& C_{26}=C_{24} \\
& C_{43}=-\frac{h}{2}\left(G_{z z} l_{z}^{2}+G_{x x} l_{x}^{2}+\frac{Q_{66} h^{2}}{6}\right) \\
& C_{27}=C_{25} \\
& C_{44}=Q_{55} h \\
& C_{28}=C_{8} \\
& C_{42}=\frac{h}{2}\left(\frac{G_{x y}\left(l_{x}^{2}+l_{y}^{2}\right)}{4}+G_{z z} l_{z}^{2}-\frac{Q_{12} h^{2}}{6}-\frac{Q_{66} h^{2}}{6}\right) \\
& C_{45}=C_{44} \\
& C_{29}=-\frac{h}{4}\left(\frac{Q_{11} h^{2}}{3}+\frac{G_{x y}\left(l_{x}^{2}+l_{y}^{2}\right)}{2}\right) \\
& C_{46}=\frac{h}{2}\left(G_{x x} l_{x}^{2}-\frac{G_{x y}\left(l_{x}^{2}+l_{y}^{2}\right)}{4}\right)
\end{aligned}
$$




$$
\begin{gathered}
C_{47}=\frac{G_{x y} h\left(l_{x}^{2}+l_{y}^{2}\right)}{8} \\
C_{48}=\frac{G_{x z} h^{3}\left(l_{x}^{2}+l_{z}^{2}\right)}{96} \\
C_{49}=-C_{48} \\
C_{50}=\frac{G_{y z} h^{3}\left(l_{y}^{2}+l_{z}^{2}\right)}{96} \\
C_{51}=-C_{50} \\
C_{52}=C_{40}
\end{gathered}
$$

